Mens

revue d'histoire intellectuelle de l'Amérique française

\title{
II. L’essor du libéralisme et du nationalisme (1815-1840)
}

Volume 6, numéro supplément, 2005-2006

URI : https://id.erudit.org/iderudit/1024237ar

DOI : https://doi.org/10.7202/1024237ar

Aller au sommaire du numéro

Éditeur(s)

Centre de recherche en civilisation canadienne-française

ISSN

1492-8647 (imprimé)

1927-9299 (numérique)

Découvrir la revue

Citer ce document

(2005). II. L'essor du libéralisme et du nationalisme (1815-1840). Mens, 6, 18-28.

https://doi.org/10.7202/1024237ar d'utilisation que vous pouvez consulter en ligne.

https://apropos.erudit.org/fr/usagers/politique-dutilisation/ 
WALLOT, Jean-Pierre (dir.), avec la collaboration de Pierre LANTHIER et Hubert WATELET, Construitions identitaires et pratiques sociales : actes du colloque en hommage à Pierre Sacard, tenu à l'Unicersité d'Ottawia les 4, 5, 6 octobre 2000, Ottawa, Presses de l'Université d'Ottawa/Centre de recherche en civilisation canadienne-française, 2002, $432 \mathrm{p}$.

\section{L'essor du libéralisme et du nationalisme (1815- 1840)}

AGEN, Marie Catherine, "The Politics of the Société Saint-JeanBaptiste de Montréal ", The American Rerien of Canadian Studies, 29, 3 (automne 1999), p. 495-510.

AJZENSTAT, Janet, «The Constitutionalism of Étienne Parent and Joseph Howe », dans Janet Ajzenstat et Peter J. Smith (dirs.), Canada's Origins: Liberal, Tory, or Republican?, Ottawa, Carleton University Press, 1995, p. 209-232.

ARBACH, Marla, Le personnage de Julie Bruneau dans la correspondance de Papineau, M.A. (Langue et littérature françaises), Université McGill, 2001, 118 p.

AUBIN, Georges, "Les femmes patriotes : de la fiction à la réalité », Bulletin d'histoire politique, 12, 1 (automne 2003), p. 43-57.

BACK, Francis, « Politique textile et comportements vestimentaires du mouvement patriote ", Bulletin d'histoire politique, 10, 2 (hiver 2002), p. 58-71.

BEAUGRAND-CHAMPAGNE, Denyse, "Orientations politiques des mouvements d'alliance et d'opposition aux Patriotes dans les comtés de Missisquoi et de Stanstead (1834-1837)», Bulletin d'histoire politique, 7, 1 (automne 1998), p. 12-18.

BELLAVANCE, Marcel, «La rébellion de 1837 et les modèles théoriques de l'émergence de la nation et du nationalisme ", Rerue d'bistoire de l'Amérique française, 53, 3 (hiver 2000), p. 367-400. 
BERNARD, Jean-Paul, «La réplique de Garneau à Lord Durham : peuple "sans histoire" ou sans avenir? ", dans Gilles Gallichan, Kenneth Landry et Denis Saint-Jacques (dirs.), FrançoisXaicer Garneau. Une figure nationale, Québec, Éditions Nota bene, 1998, p. 195-221.

BERNARD, Philippe, Amury Girod : un Suisse cher les Patriotes du Bas-Canada, Sillery, Septentrion, 2001, 255 p.

BERNARD, Philippe, "Amury Girod ou l'intellectuel militant », Bulletin d'histoire politique, 12, 1 (automne 2003), p. 90-96.

BERNIER, Marc-André, «Patriotes et orateurs : de la classe de rhétorique à l'invention d'une parole rebelle ", Voix et images, 26, 3 (2001), p. 498-514.

BOURQUE, Gilles et al., "Origines et sens de 1837. Le débat ", Bulletin d'histoire politique, 8, 2-3 (printemps-hiver 2000), p. 288-314. BRODEUR, Raymond, Catéibisme et identité culturelle dans le diocèse de Québec de 1815, Sainte-Foy, Les Presses de l'Université Laval, 1998, 309 p.

CAMBRRON, Mlicheline (dir.), Le journal Le Canadien : littérature, espace public et utopie (1836-1845), Montréal, Fides, 1999, 419 p.

CHAMIPAGNE, Monique, La question des indemnités après les Rébellions de 1837 et de 1838 dans le Bas et le Haut-Canada, M.A. (Histoire), Université du Québec à M Iontréal, 1998, 100 p.

CHARRON, Marc, "Demain tout recommence : Lord Durham's Report en traduction ", Traduction, Terminologie, Rédaction. Études sur le texte et ses transformations, 10, 1 (1997), p. 101-136.

CHUREAU, Damien, «La Maison d'industrie de Montréal (1836-1870) : l'intervention des pouvoirs publics dans l'assistance et les clivages culturels ", Bulletin d'bistoire politique, 6, 2 (hiver 1998), p. 11-18.

CIRCÉ-CÔTÉ, Ève, Papineau : son influence sur la pensée canadienne (Réédition), Montréal, LUX éditeur, 2003, 266 p. 
COATES, Colin M., «The Rebellions of 1837-1838, and Other Bourgeois Revolutions in Quebec Historiography ", International journal of Canadian Studies/Re'ue internationale d'études canadiennes, 20 (automne 1999), p. 19-34.

COLLIN, Marc, Mensonges et vérités dans les souvenirs de Félix Poutré: essai d'analyse psychohistorique d'un cas de fraude historique, M.A. (Histoire), Université du Québec à Montréal, 2002, v-171 p.

COLLIN, Marc, Mensonges et vérités dans les souvenirs de Félix Poutré, Sillery, Septentrion, 2003, 256 p.

COUILLARD, Narie et Patrick IMIBERT, «Les Déclarations de principes au dix-neuvième siècle au Canada français et leur portée dans les Amériques ", Québec Studies, 28 (1999-2000), p. 81-97.

CURTIS, Bruce A., "Arthur Buller to Msgr Signay of Quebec, 23 octobre 1839 ", Historical Studies in Education/Rerue d'histoire de l'éduiation, 13, 1 (printemps 2001), p. 49-58.

CURTIS, Bruce A., « Public Education and the Manufacture of Solidarity: Christopher Dunkin's Design for Lower Canada ", Histoire sociale/Social History, 35, 70 (2002), p. 447-468.

DESBIENS, Marie-Frédérique, «Les écrits de patriotes au Québec : circulation, conservation, diffusion et invention (18372001) ", dans Nancy Desjardins et Jacinthe Martel (dirs.), Archives et fabrique du texte littéraire, Montréal, Département d'études littéraires, Université du Québec à Montréal, 2001, p. 45-58.

DESBIENS, Marie-Frédérique, «Les Patriotes de 1837-1838: modèles et représentation, Bulletin d'bistoire politique, 12, 1 (automne 2003), p. 9-15.

DESSUREAULT, Christian et Christine HUDON, "Conflits sociaux et élites locales au Bas-Canada : le clergé, les notables, la paysannerie et le contrôle de la fabrique ", The Canadian Historical Rerien, 80, 3 (septembre 1999), p. 413-439.

DUCHARMIE, Michel, «L'État selon lord Durham : liberté et nationalité dans l'Empire britannique ", Cahiers d'histoire, 18, 2 (automne 1998), p. 39-63. 
DUCHARME, Michel, Du triptyque idéologique : libéralisme, nationalisme et impérialisme au Haut-Canada, au Bas-Canada et en GrandeBretagne entre 1838 et 1840, M.A. (Histoire), Université de Montréal, 1999, $191 \mathrm{p}$.

DUCHARME, Michel, «Penser le Canada : la mise en place des assises intellectuelles de l'État canadien moderne (1838-1840) ", Revue d'histoire de l'Amérique française, 56, 3 (hiver 2003), p. 357-386.

DUFOUR, Andrée, "Les institutrices rurales du Bas-Canada : incompétentes et inexpérimentées?", Reiue d'bistoire de l'Amérique française, 51, 4 (printemps 1998), p. 521-548.

FECTEAU, Jean-Marie, «Lendemains de défaite : les rébellions comme histoire et mémoire ", Bulletin d'histoire politique, 7, 1 (automne 1998), p. 19-28.

FECTEAU, Jean-M Larie, " "This ultimate resource..." M Iartial Law aud State Repression in Lower Canada (1837-1838) ", dans F.M. Greenwood et B. Wright (dirs.), Canadian State Trials, vol. II, Rebellion and Invasion in the Canadas (1837-1839), Toronto, The Osgoode Society fo Canadian Legal History by University of Toronto Press, 2002, p. 207-247.

FOHLEN, Claude, " "L'américanisation" du catholicisme canadien ", International journal of Canadian studies/Rerue internationale d'études canadiennes, 19 (printemps 1999), p. 151-165.

FONTAINE-BERNARD, Steven, "La France et les rébellions de 1837-1838 : connaissance et perceptions ", Bulletin d'histoire politique, 12, 1 (automne 2003), p. 125-133.

FONTAINE, Vincent, « Analyse historiographique et prosopographique des rebelles impliqués dans les rébellions de 1837-1838 dans le Haut-Canada ", Bulletin d'histoire politique, 12, 1 (automne 2003), p. 134-142.

FONTAINE, Vincent, «Les rébellions de 1837-1838 dans le Haut-Canada : récit des événements ", Bulletin d'histoire politique, 12, 1 (automne 2003), p. 116-124. 
GAGNON, Hervé, Dicertir et instruire : les musées de Montréal an XIXe siècle, Sherbrooke, GGC éditions, 1999, 241 p.

GAGNON, Serge, De l'oralité à l'écriture : le manuel de français à l'école primaire (1830-1900), Sainte-Foy, Les Presses de l'Université Laval, 1999, $236 \mathrm{p}$.

GALLICHAN, Gilles, « [ $^{\mathrm{gr}}$ Plessis et le journal de son voyage en Europe ", Les Cahiers des Dix; 54 (2000), p. 61-97.

GALLICHAN, Gilles, "La Session de 1836 ou le Parlement en grève ", Les Cahiers des Dix; 55 (2001), p. 191-294.

GAUTHIER, Viviane, Imaginer les rébellions : 1837-1838 dans le roman historique canadien-anglais et québéiois francophone au XIXe et XXe siècle, M.A. (Histoire), Université du Québec à Montréal, 2000, 163 p.

GORDON, Alan, "Heroes, History, and Two Nationalisms: Jacques Cartier", Journal of the Canadian Historical Association/Rerue de la Société historique du Canada, 10 (2000), p. 81-102.

GREENWOOD, Frank Murray et Barry WRIGHT (dirs.), Canadian State Trials, vol. II : Rebellion and Iniasion in the Canadas (1837-1839), Toronto, The Osgoode Society for Canadian Legal History by University of Toronto Press, 2002, 499 p.

GREER, Allan, "Reconsidérer la Rébellion de 1837-1838 », traduction de Denyse Beaugrand-Champagne, Bulletin d'histoire politique, 7, 1 (automne 1998), p. 29-40.

GREER, Allan, "Historical Roots of Canadian Democracy ", Journal of Canadian Studies/Reı'ue d'études canadiennes, 34, 1 (1999), p. 401-420.

HAMEL, Solange, Les patriotes oubliés de la Montérégie (1837), SaintAlphonse-de-Granby, Éditions de la Paix, 2003, 129 p.

HARDEL, Frédéric, « Lecture de Lactance Papineau, un « Canadien malheureux » (1831-1862) », dans Yvan LAMONDE et Sophie MONTREUIL (dirs), Lire au Québec au XIXe siècle, SaintLaurent, Fides, 2003, p. 95-121. 
HARDY, René, Contrôle social et mutation de la culture religieuse au Québec (1830-1930), Montréal, Boréal, 1999, 284 p.

HARDY, René, "Les fondements du renouveau religieux dans le Québec du XIXe siècle : éléments d'une réinterprétation », dans Michel Lagrée (dir.), Chocs et ruptures en histoire religieuse. Fin XI TIIeXIXe siècles, Rennes, Presses universitaires de Rennes, 1998, p. 1931.

JAMES, Kevin, The St. Patrick's Society of Montreal: Ethno-Religious Realignment in a 19th Century National Society, M.A. (Histoire), Université McGill, 1997, 91p.

JAMES, Kevin, «Dynamics of Ethnic Associational Culture in Nineteenth-Century City: Saint Patrick's Society of M Iontreal, 1834-56 ", Canadian Journal of Irish Studies/Rer'ue canadienne d'études irlandaises, 26, 1 (printemps 2000), p. 47-66.

LABRÈCHE, Christian, «De l'utilisation politique de la mémoire des Patriotes de 1837-1838 au Québec depuis la Révolution tranquille », Bulletin d'histoire politique, 10, 2 (hiver 2002), p. 150-162.

LACROIX, Laurier, "Les envois de tableaux européens de Philippe-Jean-Louis Desjardins à Québec en 1817 et 1820 : établissement du contenu ", Journal of Canadian Art History/Annales d'histoire de l'art canadien, 20, 1-2 (1999), p. 26-45.

LANONDE, Yvan, "L'Ombre du passé” : François-Xavier Garneau et l'éveil des nationalités ", dans Gilles Galichan, Kenneth Landry et Denis Saint-Jacques (dirs.), Francois-Xaicer Garneau. Une figure nationale, Québec, Éditions Nota bene, 1998, p. 51-82.

LAMONDE, Yvan, « Papineau, Parent, Garneau et l'émancipation nationalitaire (1815-1852) », Bulletin d'histoire politique, 7, 1 (automne 1998), p. 41-49.

LAMONDE, Yvan, «Le Bas-Canada et Le courrier des États-Unis de New-York (1828-1840)", Les Cabiers des Dix; 56 (2002) p. 217-233.

LAMONDE, Yvan et Sophie MONTREUIL (dirs.), Lire au Québec au XIXe siècle, Saint-Laurent, Fides, 2003, 330 p. 
LAMONDE, Yvan et Sophie MONTREUIL, « Pour une histoire des pratiques de lecture : éléments de méthode et pacte fondateur », dans Yvan Lamonde et Sophie Montreuil (dirs), Lire an Québec au XIXe siècle, Saint-Laurent, Fides, 2003, p. 7- 16.

LAMONDE, Yvan et Frédéric Hardel, «Lectures domestiques, d'exil et de retraite de Louis-Joseph Papineau (1823-1871) ", dans Yvan Lamonde et Sophie Montreuil (dirs), Lire au Québec au XIXe siècle, Saint-Laurent, Fides, 2003, p. 19-67.

LAMONDE, Yvan, "La lecture et le « livre de l'histoire » chez Amédée Papineau (1835-1845) ", dans Yvan Lamonde et Sophie Montreuil (dirs), Lire au Québec au XIXe siècle, Saint-Laurent, Fides, 2003, p. 69-93.

LAPORTE, Gilles, «Le parti patriote et les Philosophic Radicals anglais (1834-1838) », Bulletin d'histoire politique, 7, 1 (automne 1998), p. $50-65$.

LEBEL, Marc, "François-Xavier Garneau et la société de discussion de Québec », dans Gilles Galichan, Kenneth Landry et Denis Saint-Jacques (dirs.), François-Xaizer Garneau. Une figure nationale, Québec, Éditions Nota bene, 1998, p. 85-165.

LEBEL, Marc, "François-Xavier Garneau et le caractère national des Canadiens », dans Gilles Galichan, Kenneth Landry et Denis Saint-Jacques (dirs.), Francois-Xaizer Garneau. Une figure nationale, Québec, Éditions Nota bene, 1998, p. 223-261.

LEITCH, Gillian I., " "The Irish Roman Catholics of Montreal in Body Assembled": Ethnic Identity and Separate Worship in Nineteenth-Century Montreal », dans Jean-Pierre Wallot (dir.), avec la collaboration de Pierre Lanthier et Hubert Watelet, Constructions identitaires et pratiques sociales: aites du colloque en hommage à Pierre Sacard, tenu à l'Université d'Ottawa les 4, 5, 6 octobre 2000, Ottawa, Les Presses de l'Université d'Ottawa, 2002, p. 205-219.

LEMIRE, Jonathan, "La particularité du comté de Deux-Montagnes en 1837 », Bulletin d'bistoire politique, 12, 1 (automne 2003), p. 97-106. 
LE MOINE, Roger, "Philippe Aubert de Gaspé ou les affaires du "bon gentilhomme" ", Les Cahiers des Dix, 57 (2003), p. 299 321.

LINTEAU, Paul-André, "Les relations entre le Québec et les Etats-Unis depuis le XIXe siècle : le regard des historiens (19752000) ", Études canadiennes/Canadian Studies, 52 (2002), p. 21-34.

LITTLE, J.I., "Canadian Pastoral: Promotional Images of British Colonization in Lower Canada's Eastern Townships during the 1830s ", Journal of Historical Geography, 29, 2 (2003), p. 189-211.

MACKEY, Frank, Steamboat Connections: Montreal to Upper Canada (1816-1843), Montréal et Kingston, McGill-Queen's University Press, 2000, 440 p.

McNABB, Heather, Montreal's Scottish Community, 1835-65: A Preliminary Study, M.A. (Histoire), Université Concordia, 1999, 118 p.

McQUILLAN, Aidan D., "Pouvoir et perception : une communauté irlandaise au Québec au dix-neuvième siècle ", Recherihes sociographiques, 40, 2 (1999), p. 263-283.

MONETTE, Isabelle, « «L'offre de titres littéraires dans les catalogues de la librairie montréalaise (1816-1879), dans Yvan LAMONDE et Sophie MONTREUIL (dirs), Lire au Québec au XIXe siècle, Saint-Laurent, Fides, 2003, p. 201-235.

NELSON, Robert, Déclaration d'indépendance et autres écrits, 1832 1848, édition établie et annotée par Georges Aubin, Montréal, Comeau \& Nadeau, 1998, 90 p.

NELSON, Wolfred, Écrits d'un patriote, édition préparée par Georges Aubin, Montréal, Comeau \& Nadeau, 1998, 177 p.

PAPINEAU, Louis-Joseph, Un demi-siècle de combats. Interientions publiques, présentation et choix de textes par Yvan Lamonde et Claude Larin, Montréal, Fides, 1998, 666 p. 
PAPINEAU, Louis-Joseph, Lettres à Julie, texte établi et annoté par Georges Aubin et Renée Blanchet, introduction par Yvan Lamonde, Sainte-Foy/Sillery, Archives nationales du Québec/ Septentrion, 2000, 812 p.

PAQUIN, Roger, « Le curé Jacques Paquin et la rébellion des Patriotes de 1837-1838 », L'Ancêtre, 29 (automne 2002), p. 13-22.

PERIN, Roberto, «L'Église et la l'édification d'une culture publique au Québec ", Études d'histoire religieuse/Historical Studies. The Canadian Catholic Historical Association, 67 (2001), p. 261-270.

PERIN, Roberto, «Elaborating a Public Culture: The Catholic Church in Nineteenth-Century Quebec ", dans Marguerite Van Die (dir.), Religion and Public Life in Canada: Historical and Comparative Perspectices, Toronto, University of Toronto Press, 2001, p. 87-105.

PERRAULT, Louis, Lettres d'un patriote réfugié au V'ermont, 1837. 1839, textes présentés et annotés par Georges Aubin, Montréal, Méridien, 1999, $198 \mathrm{p}$.

PETRIE, Brian M., " Social Misconstructions in the Analysis of the Australian Experiences of the French-Canadian Patriote Convicts (1839-1848) », Histoire sociale/Social History, 32, 63 (mai 1999), p. 63-71.

PROULX, Jean-Pierre, «L'évolution de la législation relative au système électoral scolaire québécois (1829-1989) », Historical Studies in Education/Relue d'histoire de l'éducation, 10, 1-2 (printempsautomne 1998), p. 20-47.

RAJOTTE, Pierre, "L'horizon autobiographique du récit de voyage de François-Xavier Garneau en Angleterre et en France dans les années 1831, 1832 et 1833 ", dans Gilles Galichan, Kenneth Landry et Denis Saint-Jacques (dirs.), François-Xarier Garneau. Une figure nationale, Québec, Éditions Nota bene, 1998, p. 167-191.

RAJOTTE, Pierre, "Le récit de voyage au XIXe siècle. Une pratique de l'intime ", Globe. Rel'ue internationale d'études québécoises, 3, 1 (2000), p. 15-37. 
ROBERT, Lucie, «L'art de transformer une défaite en victoire : la représentation des Rébellions dans le théâtre de Louis Fréchette ", Bulletin d'histoire politique, 12, 1 (automne 2003), p. 16-27.

ROUSSEAU, Louis, "Crises, choc et revitalisation culturelle dans le Québec du XIXe siècle », dans Michel Lagrée (dir.), Chocs et ruptures en histoire religieuse. Fin XVIIIe-XIXe sièlles, Rennes, Presses universitaires de Rennes, 1998, p. 51-71.

SARRA-BOURNET, Michel (dir.), avec la collaboration de Jocelyn SAINT-PIERRE, Les nationalismes au Québec du XIXe siècle au XXIe siècle, [Sainte-Foy], Les Presses de l'Université Laval, 2001, $364 \mathrm{p}$.

SOSSOYAN, Matthieu, The Kahnawake Iroquois and the I onerCanadian Rebellions (1837-1838), M.A. (Anthropologie), Université McGill, 1999, 129 p.

SOSSOYAN, Mathieu, «Les Iroquois de Kahnawake et de Kanesatake et les Rébellions de 1837-1838 », Bulletin d'histoire politique, 12, 1 (automne 2003), p. 107-115.

ST-LOUIS, Chantal, "Des livres racontent : survol statistique d'un siècle d'édition littéraire au Québec (1820-1920) ", Documentation et bibliothèques, 44, 4 (septembre 1998), p. 5-13.

THIBEAULT, Marianne, «Les représentations des Patriotes dans l'art québécois : le cas d'Henri Julien », Bulletin d'histoire politique, 12, 1 (automne 2003), p. 28-42.

TRIGGER, Rosalyn, « The Geopolitics of the Irish-Catholic Parish in Nineteenth-Century Montreal ", Joumal of Historical Geography, 27, 4 (October 2001), p. 553-572.

WATT, Steven, «L'atavisme constitutionnel du gouvernement responsable », Bulletin d'histoire politique, 6, 3 (printemps-été 1998), p. 52-63.

WATT, Steven, Authoritarianism, Constitutionalism and the Special Council of Lower Canada, 1838-1841, M.A. (Histoire), Université McGill, 1998, 149 p. 
WATSON, Samuel, "United States Army Officers Fight the "Patriot War": Responses to Filibustering on the Canadian Border, 1837-1839 ", Journal of the Early Republic, 18, 3 (automne 1998), p. 485-519.

YOUNG, Brian, "The Volunteer Militia in Lower Canada (18371850) ", dans Poner, Place and Identity: Historical Studies of Social and Legal Regulation in Quebec, aites d'un colloque du Groupe sur l'bistoire de Montréal (1996), Montréal, The Group, 1998, p. 37-53.

\section{Une vie intellectuelle active : l'antagonisme libéral- ultramontain (1840-1880)}

BALMER, Randall et Catharine Randall, " "Her Duty to Canada": Henriette Feller and French Protestantism in Quebec », Churis History, 70, 1 (2001), p. 49-72.

BARRIÈRE, Mireille, «Le goupillon, le maillet et la censure du théâtre lyrique à Montréal (1840-1914) ", Les Cahiers des Dix, 54 (2000), p. 119-135.

BEAUDOIN, Réjean, « Réception critique de la littérature québécoise au Canada anglais (1867-1901) », Études françaises, 32, 3 (1996), p. 61-76.

BÉLAND, Cindy, «Salons et soirées mondaines au Canada français : un espace privé vers l'espace public », dans Pierre Rajotte (dir.), Lieux et réseaux de sociabilité littéraire au Québec, Québec, Éditions Nota bene, 2001, p. 71-112.

BÉLISLE, Jean, «Une résidence oubliée : la maison de LouisHyppolite La Fontaine ", Journal of Canadian Art History/Annales d'histoire de l'art canadien, 20, 1-2 (1999), p. 46-67. 